Supporting Information:

\title{
Cellular Responses to Exposure to Outdoor Air from the Chinese Spring Festival at the Air-Liquid Interface
}

Jie Ding, ${ }^{\dagger}, \nabla$ Jincheng Guo, ${ }^{\dagger, \nabla}$ Liming Wang, ${ }^{\S, \nabla}$ Yandong Chen, ${ }^{\dagger}$ Bin Hu ${ }^{\dagger}$ Yiye Li, ${ }^{\dagger}$ Rujin Huang," Junji Cao," Yuliang Zhao, ${ }^{\dagger}$ Marianne Geiser, ${ }^{\#}$ Qing Miao,,${ }^{\perp}$ Ying Liu, ${ }^{* \dagger}$ and Chunying Chen ${ }^{*} \dagger$

${ }^{\dagger}$ CAS Key Laboratory for Biomedical Effects of Nanomaterials and Nanosafety \& CAS Center for Excellence in Nanoscience \& Beijing Key Laboratory of Ambient Particles Health Effects and Prevention Techniques, National Center for Nanoscience and Technology of China and University of Chinese Academy of Sciences, Beijing 100190, China

*CAS Key Laboratory of Intelligent Information Processing, Advanced Computer Research Center, Institute of Computing Technology, Chinese Academy of Sciences, Beijing 100190, China

${ }^{\S}$ Division of Nuclear Technology and Applications, Institute of High Energy Physics, Chinese Academy of Sciences, Beijing 100049, China

"Key Lab of Aerosol Chemistry \& Physics, Institute of Earth Environment, Chinese Academy of Sciences, Xi'an 710061, China

${ }^{\perp}$ Divisions of Pediatric Surgery and Pediatric Pathology, Departments of Surgery and Pathology, Children's Research Institute, Medical College of Wisconsin, Milwaukee, Wisconsin 53226, United States

"Institute of Anatomy, University of Bern, 3012 Bern, Switzerland

$\nabla$ Jie Ding, Jincheng Guo and Liming Wang contributed equally to this work.

*Corresponding Authors:

Email addresses: chenchy@nanoctr.cn or liuy@nanoctr.cn or miaoq168@gmail.com

The number of pages of Supporting Information is 13 including 4 figures, and 3 tables. 
- METHODOLOGY

\section{Dynamic Monitoring of the Air Pollution during the Spring Festival}

Air pollution before, during and after the Chinese New Year was measured from the roof of a five-story building ( $15 \mathrm{~m}$ above the ground) located about $100 \mathrm{~m}$ north of the North 4 th Ring Road in Beijing (E 108.887 ${ }^{\circ}$, N 34.229 $)$. The east, west and north sides of the sampling site are residential areas and scientific research institutes. There were no factories operating nearby and almost no cars on the North 4th Ring Road during the period of Chinese New Year, though it was a major road with heavy traffic on other days (Figure 1A). Thus, fireworks and emissions from non-vehicular/non-industrial sources were likely the main sources of air pollution. This real-time exposure model suitably simulates the exposure mode of atmospheric particles at a level close to realistic human exposure.

The methods of real-time air pollution sampling were similar to those previously described ${ }^{17}$. Based on the principle of aerosol light absorption, $\mathrm{PM}_{1}$ and black carbon were measured using a multi-angle absorption photometer (MAAP-5012; Thermo Scientific), which was continuously operated at a stable airflow rate of $5 \mathrm{~L} / \mathrm{min}$ with a time resolution of 5 minutes. Ambient CO (Model ML/EC9810, Ecotech Pty Ltd, Australia), $\mathrm{NO} / \mathrm{NO}_{2} / \mathrm{NO}_{\mathrm{x}}$ (Model ML/EC9841B Analyzer, Ecotech Pty Ltd, Australia) and $\mathrm{SO}_{2}$ (Model ML/EC9850 Analyzer, Ecotech Pty Ltd, Australia) were continuously measured every 5 minutes during the sample collection periods. Conventional meteorological data (Table 1), including temperature (TP), relative humidity (RH), wind speed (WS), ambient $\mathrm{O}_{3}$ and visibility (Vis) were recorded by the China Meteorological Administration about $5 \mathrm{~km}$ south of the sampling site.

\section{Aerosol deposition chamber and cell exposure}

We employed a portable instrument for realistic toxicity testing of air pollutants, the Nano Aerosol Chamber for In Vitro toxicity (NACIVT), for short and long-term exposures with ALI cell culture. ALI is a good simulation of human exposure that the respiratory tract in 
a human will modify the PM size distribution over what is taking place in the ALI. The instrument allows the parallel exposure of up to 24 cell cultures on microporous Transwell@ inserts (polyester membrane, $0.4 \mu \mathrm{m}$ pore size, $6.5 \mathrm{~mm}$ diameter; Corning, USA). The entire chamber is temperature controlled at $37^{\circ} \mathrm{C}$. To remove non-adhering cells, culture medium was replaced before exposure. The apical cell culture medium was reduced to a residual amount $(40 \mu \mathrm{L})$ to mimic the ALI.

Exposure to $\mathrm{PM}_{1}$ was realized by the particle size cut instrument Cyclone (SCC1.197, BGI, America). After entering the NACIVT, the air was first humidified to an adjustable relative humidity $(\mathrm{RH}, 85-95 \%)$. The air was then mixed with $5 \% \mathrm{CO}_{2}$ and pumped at 0.6 L/min through the exposure chamber. Particles precipitated on the cell surfaces by electrostatic deposition. Cells in a filtered-air control group were exposed to clean air by removal of particles and gases with a mounted filter and activated carbon. The cell cultures were exposed to the air for $12 \mathrm{~h}$, either during the daytime from 8:00 am to 8:00 pm or during the night from 8:00 pm to 8:00 am of the next day. The cells were harvested immediately thereafter for subsequent assays and the conditioned media on the basal side were collected for cytokine analysis (Figure 2).

As a model for differentiated macrophages, the cell-line THP-1, derived from human acute monocytic leukemia cells, was used. Well-differentiated macrophages, in the experimental groups (E1-E5), were sequentially exposed to the aerosols for 12 hours, either during daytime (E1D1, E3D2, E5D3) or during the night (E2N1, E4N2). Thus, the exposures of E1D1, E2N1, E3D2 and E4N2 covered the period from 8:00 a.m. on New Year's Eve to 8:00 a.m. on the second day of Chinese New Year, 2015. The E5D3 exposure was performed from 8:00 a.m. to 8:00 p.m. on the fourth day of Chinese New Year. The control group was exposed to filtered air by mounting a filter and activated carbon to remove particles and gases.

\section{Particle deposition dose estimation}


The NACIVT has a total exposure flow rate of $0.6 \mathrm{~L} / \mathrm{min}$, which is equally distributed into 24 wells. We exposed the cells for $12 \mathrm{~h}$ and measured the mass concentration in real-time. The deposition efficiency was approximately $1.5 \%$, according the electrostatic deposition equipment. Transwell® insert used had a diameter of $6.5 \mathrm{~mm}$ and an area of $33.166 \mathrm{~mm}^{2}$. The total deposition concentration was calculated by the following equation:

$$
\mathrm{C}_{\text {deposition }}=\mathrm{L} \times \mathrm{C}_{\text {mass }} \times \mathrm{t} \times \mathrm{f} / \mathrm{A}_{\text {total }}
$$

Where, $\mathrm{L}$ is the flow rate, $\mathrm{C}_{\text {mass }}$ is the average particle mass concentration, $\mathrm{t}$ is the exposure time, $\mathrm{f}$ is the deposition efficiency, and $\mathrm{A}_{\text {total }}$ is the total exposure area.

\section{THP-1 Cell Culture and Differentiation}

As a model for differentiated macrophages, the cell-line THP-1, derived from human acute monocytic leukemia cells, was used. THP-1 were obtained from the American Type Culture Collection (ATCC, Manassas, VA) and cultured in RPMI 1640 media (Life Technologies) supplemented with $10 \%$ fetal bovine serum (FBS) Gold (PAA, Paschl, Austria). Differentiation was achieved by re-suspension of THP- 1 cells at $1 \times 10^{6}$ cells $/ \mathrm{mL}$ in growth medium supplemented with phorbol-12-myristate-13-acetate (PMA, 20 ng/mL), 100 units/ml penicillin, and $100 \mu \mathrm{g} / \mathrm{ml}$ streptomycin (Invitrogen, Carlsbad, CA, USA). The cells were maintained at $37^{\circ} \mathrm{C}$ in a humidified $5 \% \quad \mathrm{CO}_{2}$ atmosphere. The medium of the PMA-differentiated cells was replaced with fresh medium after $12 \mathrm{~h}$, followed by 12 more hours of incubation to establish cell stability before exposure. Well-differentiated macrophages exhibited an increase in cytoplasmic volume and firmer adherence than undifferentiated cells. And the most important role of macrophages is as the initiators of inflammatory cascades via release of signaling proteins.

\section{The miRNA microarray assay}

miRNAs were extracted using the miRNA mirVana ${ }^{\mathrm{TM}}$ isolation kit (Ambion, Life Technologies, CA, USA) and the expression of miRNA was analyzed using a TaqMan 
microRNA assay (Applied Biosystems, Foster City, CA, USA) according to the manufacture's instructions. Reverse transcription was accomplished using a polyA kit (Sangon, Shanghai, China). Briefly, $100 \mathrm{ng}$ mRNA was added to $10 \mu \mathrm{L} 2 \times$ miRNA mix and $2 \mu \mathrm{L}$ reverse transcriptase to make a $20 \mu \mathrm{L}$ final volume. The reaction was incubated at $37^{\circ} \mathrm{C}$ for $1 \mathrm{~h}$ and at $85^{\circ} \mathrm{C}$ for 5 min to inactivate the enzyme. Real-time PCR was used to quantify the amount of the different miRNAs. Each $25 \mu \mathrm{L}$ PCR reaction mixture contained $12.5 \mu \mathrm{L}$ SYBR Advantage Premix $(2 \times), 0.5 \mu \mathrm{L}$ ROX Dye (LSR $50 \times), 0.5 \mu \mathrm{L}$ miRNA-specific primer $(10 \mu \mathrm{M}), 0.5 \mu \mathrm{L}$ 3' primer, $2 \mu \mathrm{L}$ cDNA template and $9 \mu \mathrm{L}$ RNAse-free water. The qRT-PCR reactions included an initial activation step at $95^{\circ} \mathrm{C}$ for $15 \mathrm{~s}, 40$ cycles of $95^{\circ} \mathrm{C}$ for $15 \mathrm{~s}, 55^{\circ} \mathrm{C}$ for $20 \mathrm{~s}$ and a final dissociation curve at $95^{\circ} \mathrm{C}$ for $60 \mathrm{~s}, 55^{\circ} \mathrm{C}$ for $30 \mathrm{~s}$ and $95^{\circ} \mathrm{C}$ for $30 \mathrm{~s}$. U6 snRNA was used as an internal reference. The primers used are shown in Table S1.

The miRNA microarray assay was performed using a commercially available product (LC Sciences, Houston, USA). The assay began with the 3'-extension of 4 to $8 \mu \mathrm{g}$ total RNA with a poly (A) tail using poly (A) polymerase. An oligonucleotide tag was then ligated to the poly (A) tail for later fluorescent dye binding. Hybridization was performed overnight on a $\mu$ Paraflo microfluidic chip using a micro-circulation pump (Atactic Technologies, Houston, USA). On the microfluidic chip, each detection probe consisted of a chemically modified nucleotide coding segment complementary to the target microRNA (from miRBase, http://www.mirbase.org/) or other RNA (control or customer-defined sequences) and a spacer segment of polyethylene glycol to extend the coding segment away from the substrate. The detection probes were made by in situ synthesis using PGR (photogenerated reagent) chemistry. The hybridization melting temperatures were balanced by chemical modifications of the detection probes. Hybridization was performed using $100 \mu \mathrm{L} 6 \times$ SSPE buffer $(0.90 \mathrm{M}$ $\mathrm{NaCl}, 60 \mathrm{mM} \mathrm{Na} 2 \mathrm{HPO}_{4}, 6 \mathrm{mM}$ EDTA, pH 6.8), containing $25 \%$ formamide at $34^{\circ} \mathrm{C}$. After RNA hybridization, the tag-conjugating Cy3 dye was circulated through the microfluidic chip 
136 for dye staining. Fluorescence images were collected using a laser scanner (GenePix 4000B,

137 Molecular Device, Sunnyvale, CA, USA) and digitized using Array-Pro image analysis 138 software (Media Cybernetics, MD, USA). The data were analyzed by first subtracting the 139 background and then normalizing the signals using a LOWESS filter (Locally-weighted 140 Regression). The data integrity was verified with a 2100 Bioanalyzer (Agilent Technologies, 141 Santa Clara, CA). Labeled RNA was hybridized to the Agilent Human miRNA Microarray 142 (v1.0). This microarray assesses the relative expression levels of 534 miRNAs measured with 11,080 probe sets. The microarray results were extracted using the Agilent Feature Extraction 144 software.

\section{Isolation and amplification of total RNA} isolation kit (Ambion, Life Technologies, CA, USA) and reverse-transcribed into cDNA using 10 pmol oligonucleotide (oligo dT) primers and moloney murine leukemia virus reverse was performed using a commercial Master Mix (Promega) and SYBR® Green I (Invitrogen, Paisley, UK). The primers used to detect the indicated genes by qRT-PCR are shown in Table S2. The primers were designed using the Primer3 Input (http://primer3.ut.ee/), and purchased from Sangon (Shanghai, China) Beijing Branch. 

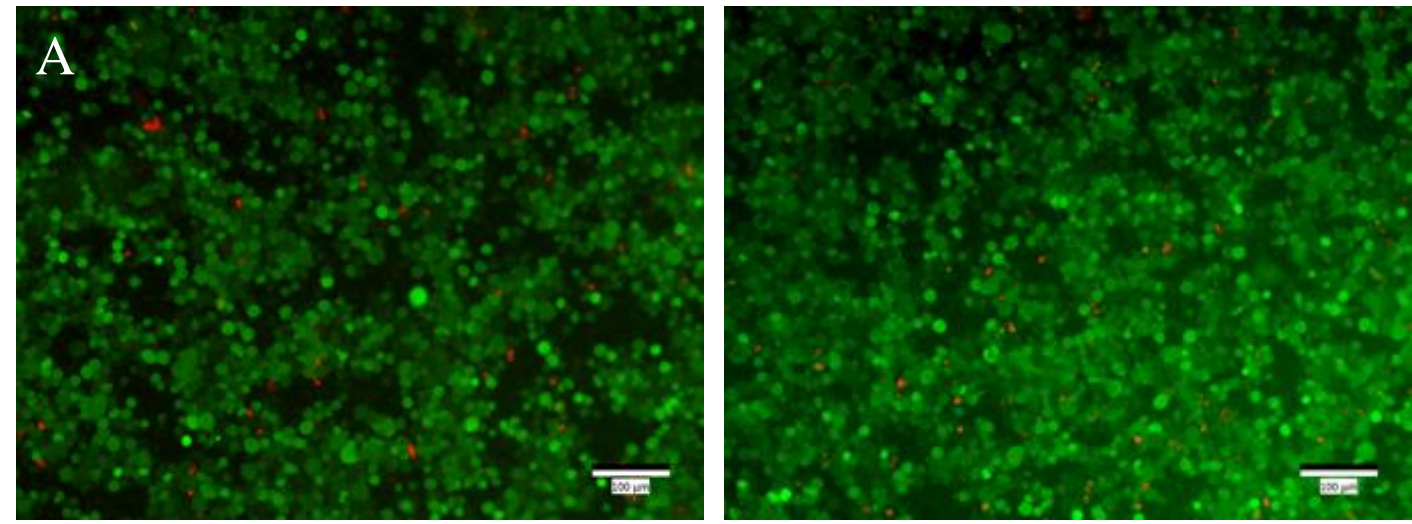

156 Figure S1. Live-dead staining of cells in the incubator control group and filter-air control 157 group. A) Cell incubator control group. B) Filter-air control group. Cells in the filtered-air 158 control group were exposed to clean air by removal of particles and gases with a mounted 159 filter and activated carbon. The growth of cells in the filtered-air control group was similar to 160 cells in the cell incubator control group. 


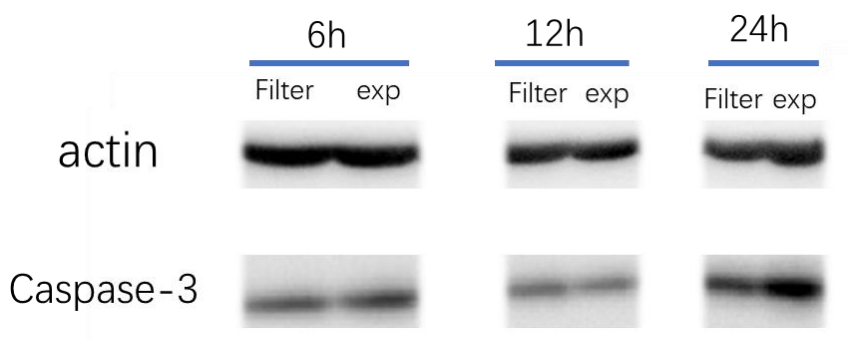

165

Figure S2. The protein expression of Caspase-3 in the incubator control group cells and 167 filter-air control group after the indicated exposure periods.

168

169 

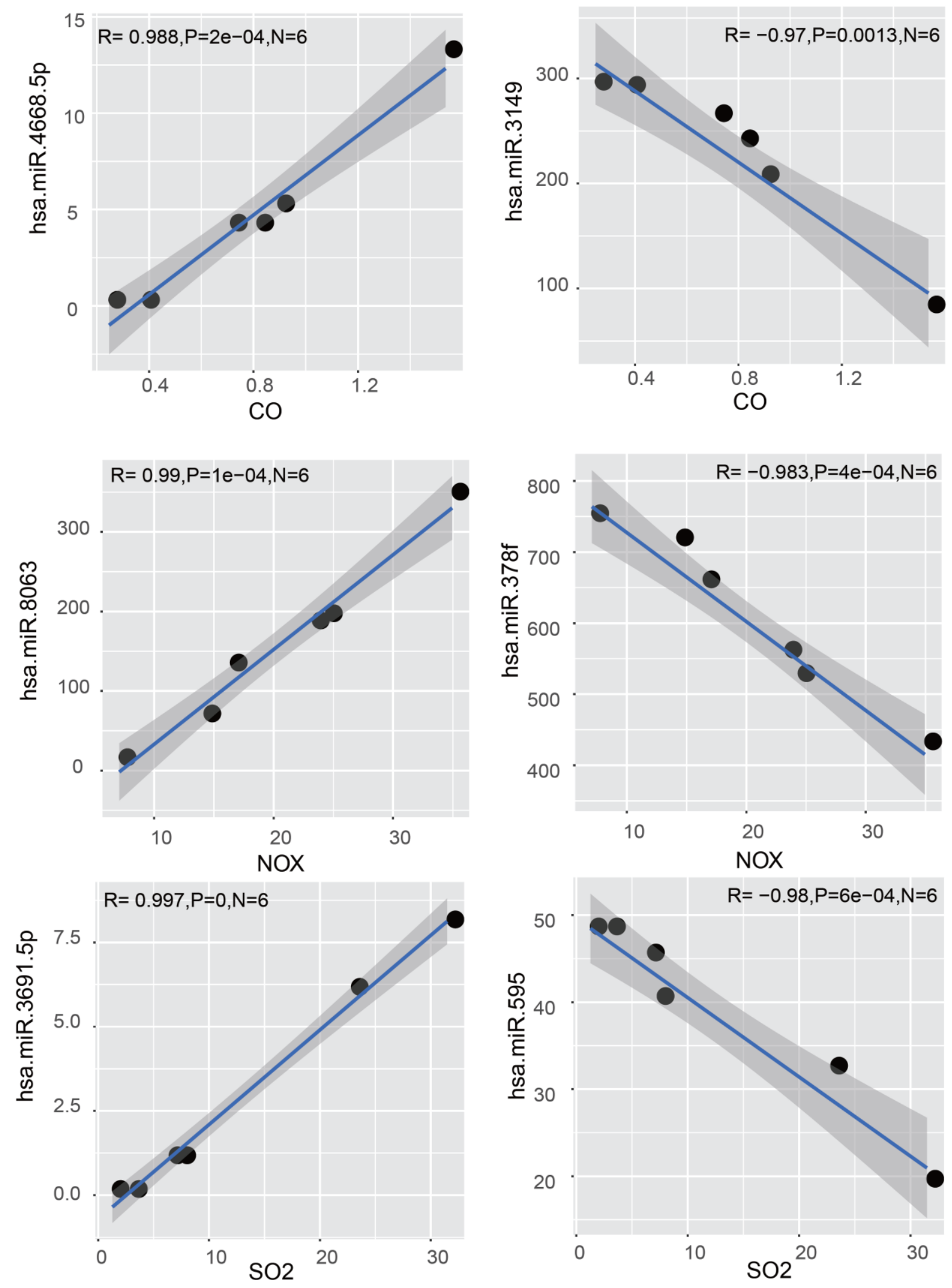

Figure S3. The correlations between microRNAs and gas pollution factor samples. 

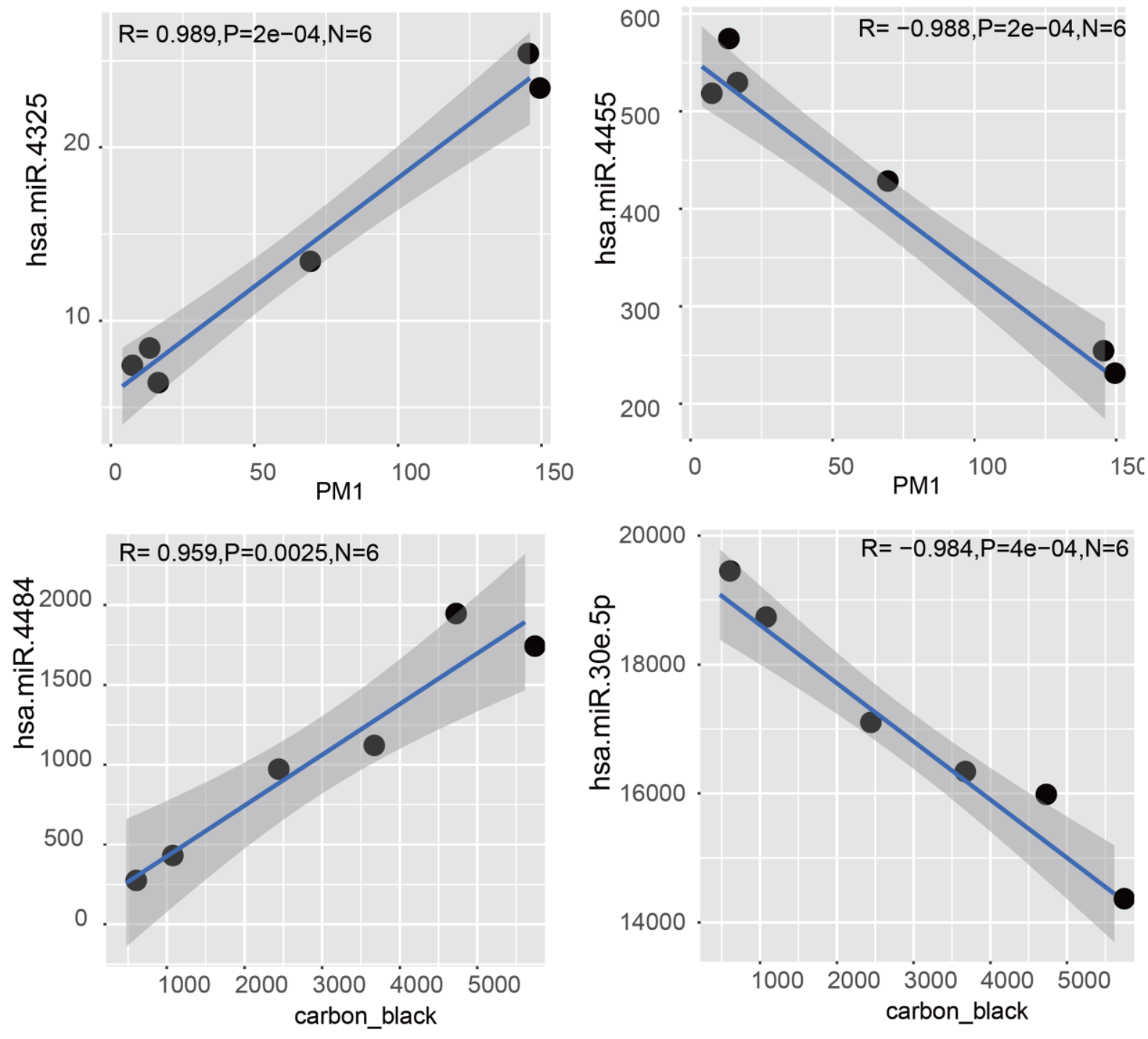

Figure S4. The correlations between microRNAs and solid pollution factor samples. 
180 Table S1. Mean values of $\mathrm{PM}_{1}, \mathrm{PM}_{2.5}$ and $\mathrm{PM}_{10}$ concentrations during the different exposure 181 periods.

\begin{tabular}{|l|l|l|l|l|l|}
\hline & E1D1 & E1N1 & E2D2 & E2N2 & E3D3 \\
\hline PM $_{10}\left(\mu \mathrm{g} \mathrm{m}^{-3}\right)$ & 17.2 & 238.8 & 254.5 & 140.2 & 185.8 \\
\hline $\mathrm{PM}_{2.5}\left(\mu \mathrm{g} \mathrm{m}^{-3}\right)$ & 13.9 & 200.6 & 217.7 & 135.5 & 31.4 \\
\hline $\mathrm{PM}_{1}\left(\mu \mathrm{g} \mathrm{m}^{-3}\right)$ & 10 & 142 & 146 & 66 & 4 \\
\hline
\end{tabular}

182

183

184 
Table S2. miRNA primers.

\begin{tabular}{|l|l|}
\hline \multicolumn{1}{|c|}{ miRNA } & \multicolumn{1}{c|}{ Forward Sequence } \\
\hline miR-155 & CGG TTA ATG CTA ATC GTG ATA GG \\
\hline miR-21 & GGC GGT AGC TTA TCA GAC TGA \\
\hline miR-30a & GCG GTG TAA ACA TCC TCG AC \\
\hline miR-125a & GGT CCC TGA GAC CCT TTA AC \\
\hline miR-125b & GCG GTC CCT GAG ACC CTA A \\
\hline let-7i & GTG AGG TAG TAG TTT GTG CTG TT \\
\hline let-7e & GCG GTG AGG TAG GAG GTT G \\
\hline miR-146b & GTG AGA ACT GAA TTC CAT AGG CT \\
\hline miR-30c & GGC GGT GTA AAC ATC CTA CAC \\
\hline miR-107 & GCG GAG CAG CAT TGT ACA GG \\
\hline
\end{tabular}


Table S3. mRNA primers.

\begin{tabular}{|l|l|l|}
\hline Gene & \multicolumn{1}{|c|}{ Forward } & \multicolumn{1}{c|}{ Reverse } \\
\hline GAPDH & 5'-CCAAGGAGTAAGACCCCTGG-3' & 5'-AGGGGAGATTCAGTGTGGTG-3' \\
\hline TLR2 & 5'-GCCAAAGTCTTGATTGATTGG-3' & 5'- TTGAAGTTCTCCAGCTCCTG-3' \\
\hline TLR4 & 5'-AGGATGAGGACTGGGTAAGGA -3' & 5'- CTGGATGAAGTGCTGGGACA-3 \\
\hline p65 & 5'-ACAAGTGGCCATTGTGTTCC-3' & 5'-ACGTTTCTCCTCAATCCGGT-3' \\
\hline IкB $\boldsymbol{\alpha}$ & 5'-CCAACCAGCCAGAAATTGCT-3' & 5'-CCAAGGACACCAAAAGCTCC-3' \\
\hline IKK及 & 5'-GAGACTCAGATCTCCCCACG-3' & 5'-TGAGCTGCTGAGACATGGAA-3' \\
\hline caspase-3 & 5'-ATGGAGAACACTGAAAACTCA-3' & 5'-TTAGTGATAAAAATAGAGTTC-3' \\
\hline caspase-8 & 5'-TGCCCAAATCAACAAGAGCC-3' & 5'-TTACCCCACACAACCTCC-3' \\
\hline caspase-9 & 5'-GCTCTTCCTTTGTTCATCTCC-3' & 5'-CATCTGGCTCGGGGTTACTGC-3' \\
\hline
\end{tabular}

